\title{
XXII. The relation between uranium and radium
}

\section{Frederick Soddy M.A. \& Thomas D. Mackenzie B.Sc.}

To cite this article: Frederick Soddy M.A. \& Thomas D. Mackenzie B.Sc. (1907) XXII. The relation between uranium and radium, Philosophical Magazine Series 6, 14:80, 272-295, DOI: $10.1080 / 14786440709463681$

To link to this article: http://dx.doi.org/10.1080/14786440709463681

$$
\text { 曲 Published online: } 16 \text { Apr } 2009 .
$$

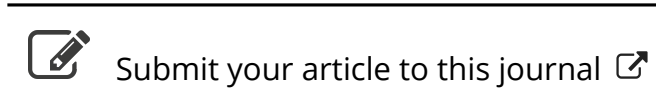

\section{Џ Article views: 2}

\section{Q View related articles $\square$}

Citing articles: 2 View citing articles 4 
spherical; one surface $a b$ [as in the annexed diagram] being convex, and the other $l c$, concave: so as to obviate any refraction of the incident and emergent conical pencils, with (any) attendant dispersion into colour within the substance of the prism.

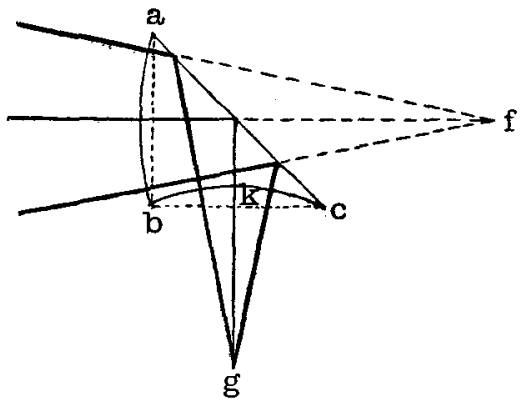

We may observe that these are not "lens-worked surfaces" in the usual acceptation of the term "lens"-which deviates or refracts rays. For these prism-surfaces are for the purpose of completely eliminating any deviation of entering and emerging rays :- securing total reflexion at $90^{\circ}$ (as desired) without dispersion.

Gross Flottbek, bei Altona, March 17, 1907.

XXII. The Relation between Uranium and Radium. By Frederick Suddy, M.A., Lecturer in Physical Chemistry and Radioactivity, and Thomas D. Mackenzie, B.Se., Carnegie Research Scholar in the Unicersity of Glasgow*.

TN a previous paper (Phil. Mag. June 1905, p. 769) one of 1 us described an experiment with a kilogram of uranyl nitrate which had been purified initially from radium by repeated precipitation of barium as sulphate in the solution, and then left for a period of 550 days to ascertain whether fresh radium was being generated in the solution. It was found at the end of the period that the amount of radium in the uranium solution had increased over a hundredfold. On the other hand, if a direct change of uranium, or of uranium $\mathrm{X}$ the first disintegration product, into radium took place, the rate of production of radium in the uranium solution should have been very much greater, it was stated about a thousand times greater, than the rate actually observed.

* Communicated by the Authors. 
The data, however, on which this latter estimate was based need some revision in the light of present knowledge, and will be again referred to in this paper. During the progress of the experiment the researcbes of McCoy (Ber. 1904, xxxvii. p. 2641), Strutt (Proc. Roy. Soc. 1905, lxxvi. A. p. 88), and Boltwood (Phil. Mag. 1905, [6] ix. p. 599) on the ratio between the quantities of the radium and uranium co-existing in the natural minerals, and the proof by the two latter that this ratio is a constant for all minerals, had established also, indirectly but quite conclusively, that uranium is the parent of radium. This satisfactory result, which is a direct prediction from the disintegration theory, can be explained in no other way, and proves, whatever the exact relationship of radium to uranium may ultimately turn out to be, that there can be no doubt that the latter produces the former.

In consequence of the extremely slow rate of production of radium from uranium which the above experiment established, the theory was put forward that one or more slowchanging transition or intermediate forms existed in the disintegration series between uranium and radium, and three or four years ago it was suggested that actinium might prove to be such a transition-form.

On the other hand, it has been pointed out (Soddy, B. A. Report, 1906, "Evolution of the Elements") that none of those hypothetical intermediate forms can exceed in period of life the original parent uranium, as the constancy of ratio with the product must hold for that parent, when there are several successive, which has the longest life in the series, and cannothold for the original parent unless it is the slowest changing of the series. Also, it is clear that the method adopted in the initial purification of the uranium from radium must be of primary importance, for the initial rate of formation of radium from any preparation of uranium will depend upon whether or not the initial purification removes, besides the radium, the intermediate parent of the latter, produced by, and therefore in ordinary circumstances present with, the uranium. Quite recently Boltwood (Am. Jour. Sci. Dec. 1906, p. 537) and Rutherford ('Nature,' Jan. 17th, 1907, p. 270; June 6th, p. 126) have observed a production of radium in preparations of actinium; and the latter has shown that this is not due to actinium itself but to a separate substance accompanying it and capable of separation. The existence of intermediate forms between uranium and radium necessarily modifies very considerably the problem. The old experiment which gave the first indication of the production of radium indicated also that uranium was certainly not the direct parent, 
274 Messrs. F. Soddy and T. D. Mackenzie on the

and left open the question whether this parent was itself formed from uranium, or was present merely as an incidental impurity derived from the mineral. It appeared that the first step to be accomplished was to purify a considerable quantity of a uranium salt by a method calculated to remove the intermediate products as well as the radium. Only in these circumstances will measurements of the initial rate of production of radium from uranium be definite. This work has occupied a considerable time, and forms the main substance of the present communication. In all about three kilograms of uranyl nitrate have been purified, and it is hoped that these preparations in the course of time will furnish the data sought, although it is already apparent that a very long period of time must elapse. For this reason it has seemed desirable to record full details of the method of preparation and testing of the solutions. The laboratory where the work has been done was new at the commencement of the work, and has been scrupulously kept from contamination with radioactive substances, so that in this respoct the conditions have been eminently favourable.

The method of purification employed in the former experiment depended on the resemblance between radium and barium, and on the fact that the latter can be removed with the former by addition of sulphuric acid. It is unlikely that this method would suffice completely to remove the intermediate products or products present in the uranium. In the present experiments the uranium was purified differently. Uranyl nitrate possesses the not at all common property of ready solubility in ether. It was considered likely that extraction with ether would free the uranium not only from incidental impurities which is known to be the case, but also from the products of its own disintegration. Sir William Crookes (Proc. Roy. Soc. 1900, lxvi. p. 409) in this way separated uranium from uranium $\mathrm{X}$ discovered by himself, and now recoguized as the first disintegration product. Our present results showed at once that the radium can also be very completely removed from the uranium by this process, and there is every reason to hope that other disintegration products are also completely removed. 'The extraction of large quantities of uranyl nitrate with ether has proved a somewhat lengthy and troublesome operation, on account of the oxidation of the ether, and the consequent formation of insoluble reduced uranium compounds. In addition several explosions have occurred, resulting in one case in the loss of some of the material undergoing purification, and in another in serious injury to one of the workers, and the loss of three 
valuable preparations of uranium $\mathrm{X}$ which had been separated and set up to be tested for the generation of radium.

The investigation here described has not been confined to the question whether radium is being produced from uranium. Special experiments have also been started to see whether uranium produces actinium, and whether actinium produces radium. Several separations of uranium X from uranium have also been made successively from the kilogram of uranyl nitrate used in the old experiment, and one is now under observation for the generation of radium. Finally, all the residues that have survived from the various extractions of uranium with ether, and other methods of purification attempted, have recently been separated very perfectly from radium, by means of barium sulphate precipitations, and are also being kept under observation for the regeneration of radium. Up to the time of writing, however, all these experiments have yielded only negative results, although in one or two of the preparations a slight increase in the content of radium is recorded, too small however to be yet certain of.

\section{Method of Testing for Radium.}

The method previously used for testing for the presence of radium by bubbling air through the solution into the electroscope, was, in the present work, employed only for preliminary tests. It gives trustworthy indications of the presence of radium, and a rough quantitative indication of the amount, but is far inferior in accuracy to the methods employed by Strutt (Proc. Roy. Soc. 1905, lxxvi. A. p. 89) and Boltwood (Am. Jour. Sci. 1904, xviii. p. 379), which are characterized by the feature that the radium emanation present is expelled from the solution by boiling. This secures the complete expulsion of the emanation, whereas only a fraction of the emanation is removed by bubbling air through the cold solution, and this fraction depends somewhat upon the conditions of the experiment.

The method employed in the present work, which however is being replaced more and more as opportunity offers by a shorter and more convenient method, consisted in the removal of the emanation from the solution by means of a mercurypump after boiling in vacuo, collecting the gas in a mercury gas-holder, and expelling it into the electroscope. The uranium solution, usually containing a kilogram of uranyl nitrate, is contained in a three-litre flask, to the neck of which is sealed a vertical all-glass condenser of the rapidcooling type. The end of the condenser is provided with a 
tap, and between this and the tap of the mercury-pump are placed a soda-lime tube and a phosphorus-pentoxide tube, separated by a tap. A side tube connects with a small bulb of 22 c.c. capacity closed at each end with taps, for admitting small quantities of air to the uranium solution. The flask is stood on the water-bath, and is heated until vigorous boiling occurs. It is then allowed to cool, a volume of air admitted and pumped out into the mercury reservoir. This process is repeated twice to ensure the complete removal of emanation. The gasholder is then connected to the electroscope through a soda-lime tube and the gas run in, a small additional quantity of air being admitted to displace the gas in the soda-lime tube. The exit and the outlet tubes of the electroscope are then closed with rubber stoppers and observations of the rate of leak taken over about half-an-hour, noting the time of admission of the gas and the time of each ohservation.

This method, which may be designated as the "pump method" in distinction to the earlier "bubbling test," has many disadvantages, the chief being that our uranium solutions tend to generate nitric oxide when heated. So long as an excess of air is present this does no harm, for it is converted into the peroxide and absorbed by the soda-lime. But the leaf-system of the electroscope has been occasionally destroyed by the formation of the peroxide in the electroscope. The method is gradually being abandoned, and that described in the section on the calibration of the electroscope used in its stead.

It is of interest to give the result of a comparison of this method of testing with the old bubbling method, in the case of a kilogram of uranyl nitrate purified by two extractions with ether. By the bubbling method, in which the uranium solution was stored in a bottle of rather over one litre capacity, with an air space of about 100 c.c. the leak in the electroscope * was found to be $0 \cdot 45$. The same solution tested by the mercury-pump method gave $4 \cdot 5$. The comparison is certainly unfavourable to the old method, as with larger amounts of radium it is probable the discrepancy would be

* Rates of leak in the electroscope throughout are expressed in divisions of the eyepiece-scale per minute, corrected for the natural leak of instrument, and unless otherwise stated, are reduced to represent the equilibrium or maximum accumulation of emanation, by means of the formula $\mathrm{L}_{\mathrm{E}}=\mathrm{L}_{\mathrm{T}} / \mathbf{1}-\epsilon_{-\lambda t}$, where $\mathrm{L}_{\mathrm{F}}$ is the equilibrium leak, $\mathrm{L}_{\mathrm{T}}$ the observed leak, $t$ the time in seconds of the accumulation of the emanation, and $\lambda$ is $2 \cdot 16 \times 10^{-6}$ (Rutherford and Soddy, Phil. Mag. 1903, [6] v. p. 447). The tables of Gruner (Jahr. Rad. u. Elekt. 1906, iii. p. 120) for the exponential function with negative exponent have proved of great use in this work. 
less. But it may be stated that the results obtained by the old methods with just detectable amounts of radium in large quantities of uranium are always several times too low.

The Electroscope.-In the present work the electroscope is the same as that previously used, although the leaf system has been frequently renewed. Here again attempts are being made to replace it by an improved form, constructed of glass silvered internally, as being more suitable for this class of work. A large number of new brass electroscopes, exactly similar to the old one, were made at the commencement of the work, but for some reason they all had a high natural leak, and the old one has been mostly employed throughout. It consists of a brass tube $10 \mathrm{~cm}$. diameter and $12.5 \mathrm{~cm}$. long provided with glass windows. The leaf system was held in a brass tube $2 \cdot 5 \mathrm{~cm}$. diameter in the centre of the upper plate. Both the upper and lower plates were of brass, and the joints were made tight with sealing-wax. It required frequent dismantling and cleaning throughout the work, for the natural leak tended to increase beyond what was thought desirable. Rubbing the inside surface of the brass with a rag moistened with dilute sulphuric acid was found more efficacious than polishing. The normal natural leak was between 0.5 and $1 \cdot 0$. The leaf system consisted of a $2.5 \mathrm{~cm}$. rubber cork coated with paraffin and carrying the charging rod made of steel wire joined to a piece of watch-spring at one end and an ebonite handle at the other. The leaf-system proper was carried centrally in the cork on a second steel wire on the lower end of which a rod of sulphur about $2 \mathrm{~cm}$. long was melted, by repeatedly dipping the end of the cold wire into just molten sulphur, and allowing a stick of sulphur gradually to build itself up on the end of the wire. Into the lower end of the sulphur was melted a brass strip $3 \mathrm{~cm}$. long and $2 \mathrm{~mm}$. broad, about $24 \mathrm{~mm}$. of the length projecting beyond the sulphur insulation. The effective length of the leaf was about $2 \mathrm{~cm}$. and the breadth $2 \mathrm{~mm}$. The microscope was that previously employed*. One division of the eyepiece scale was equal to $\cdot 0235 \mathrm{~mm}$. The scale was used in the horizontal position throughout.

It was found that the new leaf-systems constructed from time to time, although made by eye without any other measurement, varied but little in their sensitiveness. For this reason it has been thought of interest to give the full

* It was made by W. Wilson of London, and had, in addition to the horizontal and vertical movements and scales, a focussing movement, which made it perfect for the purpose of following up and keeping in focus a rapidly moving leaf. 
details of the instrument, for it suffices to follow approximately the measurements given to obtain an instrument giving without calibration a fairly accurate measure for excessively minute quantities of radium. The instrument described should certainly be trustworthy for absolute measurements of minute quantities of radium without being calibrated to within at most 50 per cent. over a range from, say, $10^{-9}$ to 10-11 gram of radium.

Calibration of the Electroscope.-The relative sensitiveness of the electroscope was determined by measuring the leak caused by the $\gamma$ rays from a constant amount of radium placed in a definite position with regard to the instrument. The radium employed was 0.2 gram of radiferous barium chloride from the Société Centrale de Produits Chimiques, of initial activity a thousand times uranium, and at the time of writing about five years old. It was placed, in its original unopened sealed glass tube, within a lead tube of wall thickness $5 \mathrm{~mm}$. and the ends of the latter were closed by hammering. This was placed $25 \mathrm{~cm}$. below the base of the electroscope, $11.4 \mathrm{~cm}$. of this distance being pine-wood (the table and block on which the instrument rested) and the remaining $13.6 \mathrm{~cm}$. air.

It seemed reasonable at the commencement of the work to anticipate that the "standard" would slowly increase in activity with lapse of time, owing to the generation of Rutherford's Radium E, but the subsequent work of Meyer and von Schweidler (Wien. Anzeiger, 12, Sitzung. April 26th 1906) and Eve (Phil. Mag. 1906, [6] xi. p. 586) has shown that no appreciable $\gamma$ radiation accompanies the $\beta$ radiation of Radium E. The most recent results of $H$. W. Schmidt (Phys. Zeit. 1907, viii. p. 361) have established the existence of such a $\gamma$ radiation, but it is so feeble, and relatively so low in penetrative power, that it cannot affect the constancy of the $\gamma$ radiation of the "standard" under the conditions described. Contrary to the initial expectation therefore the standard has probably not changed during the years the investigation has been in progress. The average leaks produced by the standard are now somewhat higher than the average two years ago, but it is impossible yet to say whether the cause is in the standard or in the electroscopes. In all, the sensitiveness of the electroscope has been determined about twenty times in the last two years, and probably with not less than ten new leaf-systems. The lowest reading recorled is $1 \cdot 8$, and the highest $3 \cdot 2$. The present mean is about $2 \cdot 7$, and this is taken as the mean sensitiveness of an electroscope constructed as described. It is remarkable 
that an indicator so excessively fragile as a gold-leaf can be reproduced to give readings so nearly ccmparable.

Such an electroscope as that described is properly charged when the leaf forms an angle with the strip of between $30^{\circ}$ and $40^{\circ}$, and no one with experience of electroscopes would use the instrument with deflexions outside these limits. It is therefore of interest to note that the values of the readings are sensibly constant over this range, and so long as the leaf is not obviously undercharged or overcharged the readings may be depended upon. In our work we aimed at a charge (about 400 volts) usually obtained from rubbed sealing-wax, such that the deflexion was $35^{\circ}$, and the divergence or horizontal distance between the tip of the leaf and the inside of the brass strip was about $11.5 \mathrm{~mm}$. at the initial charge. The potential, divergence, and sensitiveness of the leaf at various deflexions is given in the following table :-

$\begin{array}{ccc}\text { Volts. } & \text { Divergence. } & \text { Sensitiveness. } \\ 645 & 16 \cdot 2 & \cdot 72 \\ 540 & 14 \cdot 3 & \cdot 79 \\ 443 & 12 \cdot 4 & \cdot 89 \\ 385 & 11 \cdot 0 & 1 \cdot 00 \\ 354 & 10 \cdot 0 & 1 \cdot 00 \\ 289 & 8 \cdot 0 & 1 \cdot 02 \\ 260 & 7: 0 & 1 \cdot 05 \\ 188 & 4 \cdot 9 & 1 \cdot 04\end{array}$

The practical working range is represented by a bracket.

For minute leaks where the "saturation current" is easily attained, the table shows that it is better to work with a too low than with a too high charge. It may be noted that the divergence was about $.8 \mathrm{~mm}$. greater if the charging-rod was near to the leaf-system. In the above table the chargingrod was as remote from the leaf-system as possible. Of course in all measurements the charging-rod and support of the leaf-system were carefully connected to the case of the instrument and earthed.

As before (in the determinations of radium) those readings of the instrument were selected which occurred in the interval from 10 to 15 minutes after the introduction of the emanation. Although the rate of leak increases rapidly both before and after this period owing to the progressive disintegration of the emanation, the leak during the interval is always constant and constitutes a temporary maximum which is a far more convenient point to select than the true maximum which is reached after some hours. It is difficult to ensure that an electroscope, which is frequently being dismantled and cleaned, 
is always perfectly air-tight, and in all our later measurements the air-tightness of the instrument has been tested immediately before each test. A small leak which may exert no appreciable effect in 10 minutes might have an important influence in several hours. In point of gain of time, both in the actual measurement and the quicker recovery by the electroscope of its normal natural leak after the emanation is blown out, the advantages of the short method of working are very great. The curve shows the period of temporary maximum after 10 minutes very clearly. It was obtained in an electroscope of sensitiveness $3 \cdot 2$ to the $\gamma$-ray test, by the introduction of the emanation re-accumulating in six days in a solution of 3.5 milligrams of uraninite. This curve is essentially the inverse of the decay-curve of the excited activity of radium produced by short exposure to the emanation (Rutherford, 'Radioactivity,' 1905, p. 307).

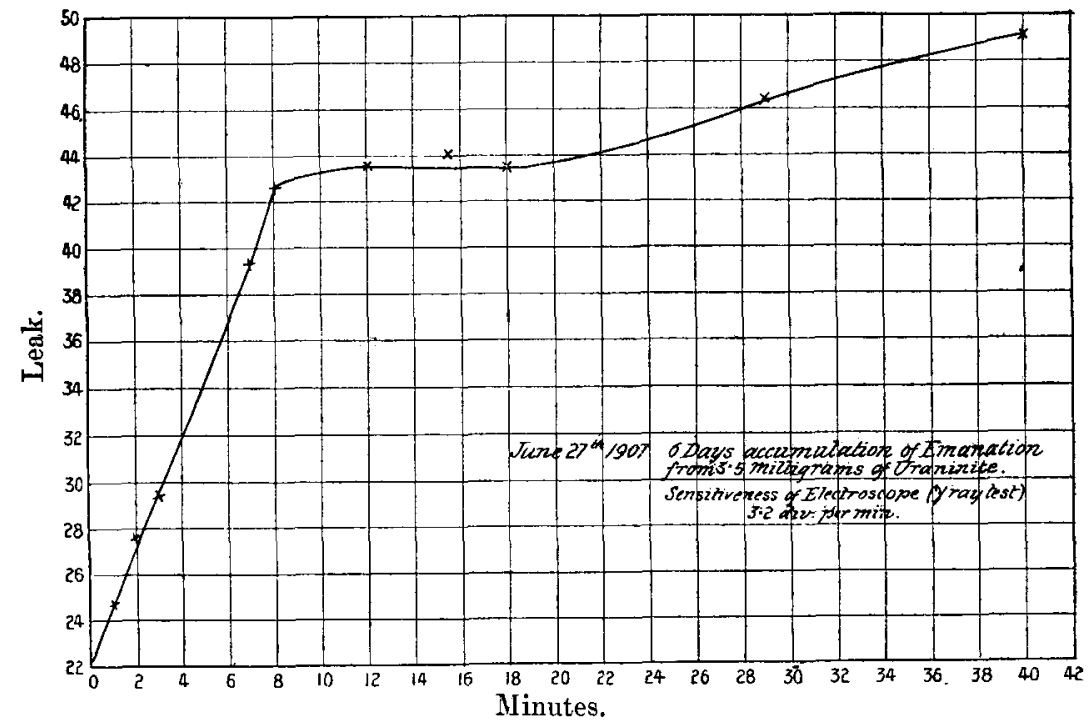

In the previous work the instrument was standardised in terms of radium by direct comparison with a known small fraction of the emanation from a weighed small amount of pure radium bromide, and it was stated that a leak of 1 . corresponded to the emanation from about $10^{-10}$ gram of radium. The new method of calibration based on the work of Boltwood and Rutherford (Am. Jour. Sci. 1906, xxii. p. 1), and used by the former, was employed in the present work. The radium is weighed ont as uraninite, a sample of which is 
analysed and the content of uranium determined. The quantity of uranium present multiplied by $38 \times 10^{-6}$ (the constant ratio of radium to uranium in minerals) gives the quantity of radium in the uraninite used, and in this way quantities of radium of the order of $10^{-9}$ gram may be weighed out directly.

The uraninite is introduced into a flask of about 100 c.c. capacity, in the neck of which bulbs are blown, and a small tube also blown into bulbs is sealed to the neck to act as a trap and prevent loss of the solution by bumping during boiling. The flask is exhausted and a quantity of aqua-regia drawn in and the neck sealed. When solution is complete the seal is broken under a clamped rubber tube and a quantity of water is drawn in. The emanation is extracted by connecting the rubber tube to a small gas washing-bottle, to serve as a trap, and a larger similar bottle, both previously exhausted on the water-pump and closed. Between the flask and the first bottle is a T-tube connected to a water-supply. The uraninite solution is boiled vigorously on the water-bath, until all gas is expelled, and it is then removed and sealed up for the next test. The gas expelled is driven into the larger of the two bottles by flowing in water, and in the same way sent into the electroscope through the soda-lime tube, the gas in the latter being displaced as before by a small additional volume of air.

This method of working is so convenient and rapid that it is being gradually used in all the tests. The preparations sealed to the mercury pump are, as opportunity offers, being cut down and transferred to flasks with inlet and outlet tubes sealed into the neck. The tubes are kept sealed, and are broken under rubber before, and resealed after, each test. As already mentioned, very similar methods have been described by Boltwood and Strutt. The possible objection to the method, that some of the emanation may redissolve in the water used, must be tolerated in riew of its manifold advantages over the other methods tried. The error, if any, is likely to be but small.

The uraninite employed was pitchblende from Joachimsthal, of activity 4.2 times uranoso-uranic oxide. The activity was determined by sprinkling some $10 \mathrm{mg}$. of the oxide and some $4 \mathrm{mg}$. of the finely powdered mineral, on two similar metal disks $2.5 \mathrm{~cm}$. in diameter, and comparing the leaks produced in an electroscope. An analysis of the mineral showed the presence of 53 per cent. of uranium. Each milligram of the mineral therefore contains $0.53 \times 0.38 \times 10^{-9}=2 \times 10^{-10} \mathrm{gram}$ of radium. 'The first test with the solid mineral immediately

Phil. Mag. S. 6. Vol. 14. No. 80. Aug. 1907. U 


\section{Messrs. F. Soddy and T. D. Mackenzie on the}

after solution must be corrected for the escape of emanation from the powdered mineral. An experiment showed that about 8.7 per cent of the emanation generated escaped from the powdered mineral, so the first tests are corrected for this amount.

It was not known whether the result obtained with a reaccumulation of emanation in the uraninite solution could be relied upon owing to the possibility of the radium precipitating out in a non-emanating form after solution of the mineral, and the experiments included an examination of this point. It was found that the results with the emanation in the solid mineral, corrected for the escape as indicated, agreed satisfactorily with later ones from the same sample of mineral in solution, so that it would be easier in calibrating an electroscope to dispense altogether with the initial tests with the solid and the necessity of determining the rate of escape from the solid, and to start with a known period of reaccumulation after boiling out the emanation from the dissolved mineral and sealing up the flask. The following Table shows the results obtained with three different quantities of mineral :-

\begin{tabular}{|c|c|c|c|c|c|c|c|c|}
\hline & Daie. & $\begin{array}{l}\text { Milligrams } \\
\text { Uraninite. }\end{array}$ & $\begin{array}{c}\text { Observed } \\
\text { leak per } \\
\text { milligram. }\end{array}$ & $\begin{array}{c}\text { Time of } \\
\text { accumu- } \\
\text { lation. }\end{array}$ & $\begin{array}{l}\text { Colrected } \\
\text { leaks per } \\
\text { nilligram. }\end{array}$ & $\begin{array}{c}\gamma \text {-ray test } \\
\text { of Electro- } \\
\text { scope. }\end{array}$ & \multicolumn{2}{|c|}{$\begin{array}{l}\text { Teak in } \\
\text { Electro- } \\
\text { scope of } \\
\text { mean sensi- } \\
\text { tiveness. }\end{array}$} \\
\hline $1 \ldots$ & $14 / 6 /-07$ & 13.5 & 133 & Solid & $14 \cdot 6$ & $\ldots$ & $\ldots$ & \\
\hline $2 \ldots$ & $19 / 6 /-07$ & $3 \cdot 5$ & $10 \cdot 7$ & , & $11 \cdot 7$ & $\ldots$ & $\ldots$ & \\
\hline $3 \ldots$ & $26 / 6 /-07$ & $2 \cdot 9$ & 135 & $"$ & $14 \cdot 3 *$ & $3 \cdot 2$ & $12 \cdot 0$ & \\
\hline $4 \ldots$ & $27 / 6 /-07$ & 35 & $12 \cdot 25$ & 6 days & $18 \cdot 1$ & $3 \cdot 2$ & $15 \cdot 3$ & Mean \\
\hline $5 \ldots$ & $10 / 7 /-07$ & 29 & $12 \cdot 8$ & 1375 days & $13 \cdot 8$ & 285 & $13 \cdot 0$ & \\
\hline $6 \ldots$ & $10 / 7 /-07$ & 35 & $15 \cdot 3$ & 13 days & $16 \cdot 8$ & $2 \cdot 7$ & $16 \cdot 8$ & \\
\hline
\end{tabular}

* In this test the solid was sealed up in the flask for two days before the test, and this is allowed for in the correction.

The mean of the last four results gives $14: 3$ per milligram in an electroscope of mean sensitiveness, so that a leak of 1 corresponds to $1.4 \times 10^{-11}$ gram of radium. The minimum detectable quantity of radium in any individual experiment was never less than one-half and usually about one-fifth of this amount.

The earlier result obtained by the use of radium directly gave $10^{-10}$ gram of radium as the equivalent of 1 division per minute, and this is therefore probably about seven times too great. It will be recalled that there was an error in the 
same direction in the early determination by Rutherford and Boltwood of the ratio of uranium to radium in minerals, which involved the comparison of the emanation produced by known small quantities of radium and by uraninite. It was ascribed by them to the partial precipitation of the radium in the standard solution, and caused the value first given for the ratio of radium to uranium to be twice too great.

At this stage the data given in the last paper may be profitably reconsidered. In the old experiment the radium emanation from the kilogram of uranyl nitrate gave a leak in the electroseope of 15 divisions after 550 days. This corresponds according to the new calibration with $2 \times 10^{-10} \mathrm{gram}$ of radium instead of $1 \cdot 6 \times 10^{-9}$ as before stated. But owing to the low value probably given by the bubbling test, this is likely to be if anything too low.

There is a discrepancy in the theoretical estimates of the rate of disintegration of uranium according to the method employed in the calculation. The value in the last paper for the fraction disintegrating, $6 \times 10^{-17}$ per second $=1.9 \times 10^{-9}$ annually, was derived from a consideration of the relative a-ray activity of uranium and radium, and the relative number of $\alpha$-ray changes in each. This was the only method then available, but neither factor was or is very certainly known. The work of Boltwood and Rutherford on the ratio of the amount of uranium to that of radium in equilibrium in the natural minerals, gives directly the ratio of the rate of change of radium $\left(2 \cdot 6 \times 10^{6}\right)$ in terms of that of uranium as unity. Rutherford takes $2 \times 10^{-10}$ (year) ${ }^{-1}$ as the fraction of uranium disintegrating, which is about ten times smaller than the value before used. It is to be remarked that this value makes the equilibrium $\alpha$-ray activity of radium about 15 million times that of uranium, assuming only one a-ray change in the latter case and four in radium*. Possibly it may turn out that more than $\alpha$-ray change is concealed in the change of uranium into uranium $\mathrm{X}$.

Accepting Rutherford's value in 500 grams of uranium in 550 days $1.5 \times 10^{-7}$ gram disintegrates, instead of $1.5 \times 10^{-6}$, the old estimate. The result is a rate of production of radium 700 times less, instead of, as stated, a thousand times less, than that calculated on the assumption of a direct change of uranium into radium. Owing to the fact that the solution was not boiled this estimate is necessarily somewhat indefinite.

* Thus Boltwood (Am. Jour. Sci. 1906, xxi. p. 409) states that the final or equilibrium activity of radium is 5.64 times the initial, and Bragg (Phil. Mag. 1906, [6] xi. p. 148) has shown that the range, and therefore the ionizing power, of the $\alpha$ particle from radium is the same as that from uranium. Hence the $a$-ray activity of radium in equilibrium ought to be $5.64 \times 2.6 \times 10^{6}=14.6 \times 10^{6}$ times that of uranium. 


\section{Puritication of the Uranium.}

The uranyl nitrate, usually one kilogram, dissolved in about one litre of water was thoroughly shaken in a bottle of 3 litres capacity with an equal volume of ether to expel air, the stopper then inserted and tied down. The bottle was fastened in the shaking machine and shaken for an hour: The liquid was allowed to stand for at least an hour to allow the two solutions to separate, and the top etbereal solution drawn off as thoroughly as possible by means of a siphon into a separating funnel and allowed to stand in the funnel for two hours when any separated aqueous liquid was removed. To the aqueous solution in the bottle a further quantity of ether was added, the mixture shaken and separated, and then a third quantity was added and treated in the same way. Three successive extractions removed nearly the whole of the uranium, and the aqueous liquid was worked up as residues. If expectations are fulfilled these residues should contain all the intermediate parent of radium.

The ethereal uranium solution was separated from ether by distillation. The usual precautions were taken in distilling ether, the distilling-flask being kept about two-thirds full by running in fresh solution as the ether evaporated. During the first distillation the uranium nitrate in the flask was allowed to collect in fairly large quantity, and owing to a sudden evolution of oxides of nitrogen a violent explosion occurred and much of the uranium was lost. In two subsequent distillations an explosion was avoided by rapidly cooling the flask as soon as gas began to appear. Thereafter, about 100 c.c. of water was kept in the flask and the uranium nitrate not allowed to collect in large quantity. The contents of the flask were poured out into a porcelain dish and heated on the steam-bath. When the liquid got hot a sudden rapid evolution of oxides of nitrogen took place lasting two or three minutes. About 50 c.c. of concentrated nitric acid were added, and the solution evaporated till a crust of erystals formed. The solution was diluted and allowed to stand, when a copious yellow precipitate, containing uranium and organic matter, was thrown down. This was separated and ignited, and the uranium oxide dissolved in nitric acid and added to the rest of the solution. This solution constituted the purified uranyl nitrate solution used in the experiments.

An attempt to avoid decomposition of the ether by the use of the purest ethyl ether obtainable met with no success. The same strong smell of aldehyde and other bodies was 
observed, the yellow precipitate formed as before, and the liquid gave off oxides of nitrogen freely. In the first purification in Experiment II. the first extraction was made on the solid crystals as supplied by the maker, and in the other purification in this experiment and those in Experiment III. the crystals obtained by evaporating the solution to dryness were employed in the first extraction.

The residues were worked up by evaporation with concentrated nitric acid, and then diluted and purified from radium as described under Experiment VII. Owing to accidents and various other causes, these residues do not represent the whole of the three kilograms employed. The residues from the old kilogram (Experiments III. to V.) contained barium and were unfortunately rejected. Probably the greater portion of the residues from the other two kilograms is present.

\section{Experiment I.}

This experiment is the one that has been longest started of those with the new method of purification. A kilogram of commercial uranyl nitrate was employed, and in its original state when tested for radium by the bubbling test produced a leak of 190 in the electroscope, equivalent to between $10^{-8}$ and $10^{-9}$ gram radium. On June 20th, 1905, after the first purification with ether a bubbling test gave a leak of $5 \cdot 5$. After a second purification in the same way it gave (July 3rd, 1905) a leak of 0.4 , and on Sept. 12th, 1905, 0.45, both by the bubbling test. Two tests performed by the mercurypump method gave (Oct. 3rd, 1905) 4.7, and (Oct. 10th, $1905) 5 \cdot 5$.

At this stage the solution contained by analysis 280 grams of uranium (element). The solution was purified a third and last time with ether and finally set up in a closed flask connected to the mercury pump on October 24th, 1905. Unfortunately the record of the final analysis has been mislaid, but it may be assumed for present purposes that about 250 grams of uranium is present. The determination will be done again at the first opportunity. The first test in the series of measurements of the quantity of radium present, which has been continued uninterruptedly since, was done on October 31st, 1905 , and the leak obtained was $1 * 4$.

In the table (p. 286) the results up to date are collected. It has been thought advisable to record every time the gas has been pumped out of the flask even though the test was a failure. 
TABLE.--Experiment I.

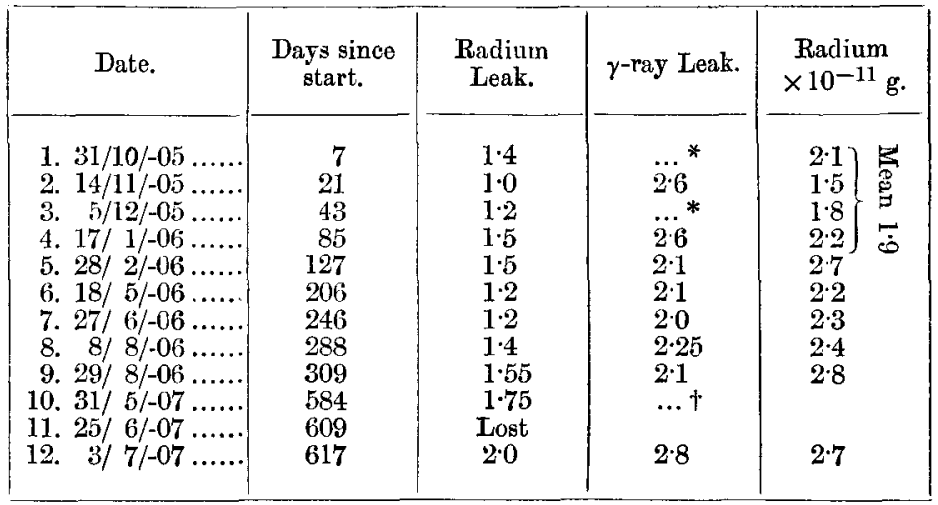

* It is probable that the $\gamma$-ray leak of the electroscope in tests Nos. 1 and 3 was similar to that found in tests Nos. 2 and 4 , but it was not actually taken.

+ Through a mistake the $\gamma$-ray leak in this 10 th test was inaccurate and it has been omitted.

Regarding first the results of the actual observations recorded in the third column, it will be seen that the low est result is the second, 21 days from the start, and the highest, which is just twice the lowest, is the last, 617 days from the start. For the last six tests there has been a regular increase from $1 \cdot 2$ to $2 \cdot 0$, and the greater uniformity of the latter half of the test may be the result of the experience gained in the former tests. The values corrected for the sensitiveness of the electroscope as shown by the $\gamma$-ray test in the fourth column, are recorded in terms of $10^{-11}$ gram of radium in the last column, but these results are not so regular as the actual observations. It is to be noted that a gradual increase in the value of the $\gamma$-ray standard with time would operate against and tend to mask a real increase in the quantity of radium present.

The long interval of 275 days between the ninth and tenth tests was intentionally allowed, so that this experiment might be as nearly as possible comparable, in point of lapse of time between the tests, with the former experiment. Considerations similar to those discussed in the next experiment made it very desirable to prove that the positive result before obtained was not due to the long interval that elapsed between the tests at the end of the first year from purification.

The results may be summed up by the statement that the amount of radium produced from 250 grams of uranium in 600 days is too small to be sure of. It is probable that the slight increase observed may prove to be real, but more time 
must elapse before this can be decided. If we take the mean of the first four observations as giving the quantity of radium initially present, this is $1.9 \times 10^{-11} \mathrm{gram}$, and the subsequent increase is therefore not greater than $10^{-11} \mathrm{gram}$.

Accepting Rutherford's value for the rate of disintegration of uranium in 250 grams in 600 days $0.8 \times 10^{-7}$ gram of uranium disintegrates. The amount of radium formed is therefore not greater than one eight-thousandth part of the uranium disintegrating.

In this connexion may be mentioned an experiment of Boltwood (Am. Jour. Sci. 1905, vol. xx. p. 239) who kept a quantity of 100 grams of uranyl nitrate ( $=48$ grams of uranium), purified by repeated crystallization from water, for 390 days and was nnable, either initially or finally, to detect any radium present. It was stated that $1.7 \times 10^{-11}$ gram of radium could have been detected if present. Estimating as before, the amount of uranium disintegrating is $10^{--8}$ gram, about 600 times the quantity of radium stated to be detectable. The experiment is with too small a quantity of material yet to afford much information. It corresponds to a period of less than six weeks with a kilogram of material. It is clear that repeated crystallization from water would have a better chance of removing intermediate products than precipitation with barium sulphate, but even if it had not done so the effect sought, with the small quantity of uranium in the time of the experiment, could only have been but small. On the basis of the old experiment with a kilogram of material, which gave a leak of 15 in 550 days, 100 grams in 400 days would give a leak of 1 , of the same order as the natural leak of our electroscope, and as the limit, $1.7 \times 10^{-11}$ gram of radium, detectable. Making allowance for this limit as stated being too high (Rutherford and Boltwood, Am. Jour. Sci. 1906 , vol. xxii. p. 1), and for the fact that Boltwood boiled his uranium solution, it is probable that a growth of radium, if it had occurred at the same rate as in the sample purified by barium sulphate, would have been detectable but only by a narrow margin.

\section{EXPERIMENT II.}

This experiment is practically a duplicate of the last and was designed to cover the possibility of one of the intermediate disintegration products between uranium and radium being a gas with considerable life-period, not itself radioactive but disintegrating raylessly, ultimately generating radium. In Experiment No. I. such a gas would be removed in the periodic tests and lost, and the result on this account might 
be too low or negative. In this experiment it is intended to carry out tests but rarely, and to preserve the gas pumped out.

A fresh kilogram of uranyl nitrate from the manufacturer was purified twice by extraction with ether, and first tested on Nov. 28th, 1906. It gave a leak, by the pump method, of $2 \cdot 8$, and was therefore cut down and again purified with ether. It was finally set up in the closed flask connected with the mercury pump on December 13th, 1906. Tested on January 10th, 1907, with an electroscope giving 1.87 to the $\gamma$-ray test, it gave a leak of only 0.64 , equivalent to $1.3 \times$ $10^{-11} \mathrm{~g}$. radium. This very satisfactory result is perhaps to be ascribed to the yellow precipitate before mentioned not being worked up and added to the solution in the last purification but added to the residues. An analysis of the solution showed the presence of 481 grams of uranium. It was first pumped out after 56 days on March 7 th, 1907, and the gas was introduced and stored in a special electroscope of about 50 c.c. volume, constructed out of glass silvered internally. The leaf system has been kept positively charged in the hope that if a solid is deposited, it may follow the usual rule and seek the negatively charged glass rather than the leaf system, which may want renewal in the course of time. The original natural leak of the electroscope was 0.12 in air, and 0.11 in a vacuum. The addition of the gas from the uranium increased the leak by 0.13 at first and 0.06 after three days. The last test was done on June 13th, 1907, after the gas had been in the instrument 98 days, and the total leak was then only $0 \cdot 09$, rather less than the natural leak initially.

The gas was again pumped out from the uranium on June 29th, but it was too large in volume for the second electroscope prepared for it owing to air having leaked in. It is at present being stored in a sealed glass tube while a larger electroscope is being constructed. A tost for radium in the solution was done on July 9th, 1907, 208 days from purification, and showed a leak of 0.9 in an electroscope with $\boldsymbol{\gamma}$-ray leak of $2 \cdot 8$.

This is equivalent to $1 \cdot 23 \times 10^{-11}$ gram of radium, which is practically identical with the initial test. The growth of radium in this experiment cannot exceed $5 \times 10^{-12}$ gram. The amount of uranium estimated to have disintegrated is $5 \cdot 3 \times 10^{-8}$; so that the production of uranium is here below the ten-thousandth part of the uranium disintegrating. Owing to the much larger amount of uranium and the much smaller initial amount of radium in this experiment than in the last, it constitutes a considerable improvement, and although 
started much later it already furnishes information equally advanced to that in the first experiment. For the future it will be much the more valuable of the two.

It should be mentioned that to guard against the unknown gas being absorbed by the mercury of the pump a side-tube containing mercury was sealed on the flask. The soda-lime and phosphorus-pentoxide tubes are not rejected when used up, but sealed up and kept for future examination.

\section{ExPERTMENT III.}

The first disintegration product of uranium is known to be uranium X, discovered in 1900 by Sir William Crookes. Its period (in which one-half cbanges) is 22 days, and in this time a uranium solution after purification regenerates one-half of the equilibrium quantity of new uranium $X$. The experiment consisted in separating the uranium $\mathrm{X}$ regenerated by a solution of uranium in the course of some weeks after purification and setting it up on the mercury pump to be tested for the growth of radium. Such a growth is theoretically to be expected, but it must necessarily be much less than in the case of uranium itself, in which there is a steady production of new uranium $\mathrm{X}$ replacing that disintegrating.

The kilogram of uranyl nitrate employed was that used in the former experiment and contained an excess of barium nitrate. It was extracted with ether, which Crookes found completely separated the uranium $\mathrm{X}$, and tested for radium on Dec. $16 \mathrm{th}, 1905$, fifty-one days from purification. The leak was $5 \cdot 1$ in an electroscope giving $2 \cdot 6$ to the $\gamma$-ray test. An attempt made to separate the uranium $X$ reproduced in this interval by the ammonium carbonate process of Crookes failed with the large quantity on account of the insolubility of the uranium precipitate. The ammonium salts were removed by evaporation with nitric acid, and ignition in small quantities. An attempt was then made with Becquerel's recently published method of removing uranium X (Compt. Rend. 1905, vol. cxli. p. 485), and the uranium-nitrate solution was boiled with, in all, eleven successive quantities of Kahlbaum's charcoal. This reduced the $\beta$ radiation, and therefore the uranium $\mathrm{X}$ present, to 20 per cent. of the initial, and further boiling with three successive quantities of lanıp-black did not reduce this amount. Unfortunately these uranium $X$ preparations were lost in the explosion before mentioned.

Ultimately the ether-extraction process was adopted. On June 18th, 1906, after the uranium solution had been kept for 
three months, the extraction was commenced. The aqueous residue was concentrated and re-extracted till nearly all the uranium was removed. It was then precipitated with ammonium carbonate in excess, and the small precipitate containing the uranium $\mathrm{X}$, as shown by a test on a photographic plate, was sealed up in a flask on June 26th, 1906 . It was tested for radium on July 9 th, in an electroscope giving 1.9 to the $\gamma$-ray test, and gave a leak of 6.5 , equivalent to $13 \times 10^{-11}$ gram radium. It is possible, in the numerous operations with the uranium since the last test, that a trace of the radium-containing residues from earlier work contaminated the quantity, which would account for the somewhat high result.

This preparation was left until June 20th, 1907, but the gas pumped out on that date was lost. On July 1st, 1907, another test was made in an electroscope giving $2 \cdot 68$ to the $\gamma$-ray test, and gave a leak of 10.5 , equivalent to $15 \times 10^{-11}$ gram of radium. It is possible that this slight increase in the quantity of radium during the 370 days the experiment has been in progress is real, and due to a partial separation of the hypothetical parent of radium with the uranium $\mathbf{X}$, but in view of the comparatively large amount of radium present it is at present very doubtful, and further results must be awaited.

\section{EXPERIMENT IV.}

This experiment and the next have to do with the possibility that actinium is an intermediate member in the uraniumradium series. An attempt has been made to detect the growth of actinium in the uranium solution employed in the last two experiments. Actinium was tested for by passing a rapid stream of air for two hours through the uranium solution boiling under reduced pressure in a flask with upright condenser placed on the water-bath. After the first half-hour the air was passed into a brass cylinder provided with a central insulated and removable electrode charged negatively to 250 volts with respect to the cylinder. The volume of the condenser was 50 c.c., and the rate of the air-stream 85 c.c. per minute measured at atmospheric pressure. The pressure was kept at about $100 \mathrm{~mm}$. of mercury, and it was estimated that the time taken for the gas to pass from the uranium solution into the neighbourhood of the negative electrode was not greater than 5 seconds, and probably less. So that a fraction of somewhat less than one-half of any actinium emanation generated would deposit its products upon the electrode. The latter was coiled up and tested in the electroscope before 
and after the test, and an increase in the rate of leak of the electroscope looked for. The initial test for actinium was carried out on August 31st, 1906, 17 days from the purification, but no evidence of the presence of actinium was obtained. Nine subsequent tests have been made at intervals, the last being done on July 1st, 1907, 304 days from the purification. Usually there has been a very slight temporary increase in the leak of the electroscope with the wire after the experiment, compared to the leak before, but it has not yet exceeded in any case the possible error. In all cases of course the measurements have been continued to see if the added increase decays with time, and in this way the possible error due to the contamination of the electrode with uranium during the test has been eliminated.

On June 5th, 1907, a test for actinium in the uranium solution was made by simply exposing overnight a negatively charged wire near the surfuce of the solution contained in a large open beaker lined inside with tinfoil kept positively charged with respect to the wire. The result was the same as previously.

The uranium solution contains 278 grams of uranium (element).

The amount of actinium detectable is necessarily much greater than in the case of radium. The period of actinium A, the longest-lived of the disintegration products of actinium used in the detection of the latter, is about 170 times less than that of the radium emanation, and for similar quantities of the parent elements the amount of disintegration products accumulating, and therefore the effect sought, must be 170 times less in the case of actinium than in that of radium. This is on the assumption that the $a$ particle from the actinium series is as efficient an ionizer as that from the radium series. Under the most favourable circumstances only a fraction of the actinium emanation generated can be made to produce actinium $A$ on the wire used in the test. As a rough estimate, the amount detectable may be taken to be not less than a thousand times greater than in the case of radium. This is about $3 \times 10^{9}$ gram. In 278 grams of uranium in 300 days $4 \cdot 6 \times 10^{-8}$ gram of uranium disintegrates.

\section{Experiment V.}

If actinium is the parent of radium it is to be expected that the actinium emanation should, like radium itself, leave behind a long-lived feeble residual activity after the ordinary excited activity has disappeared, and this should prove to be 
or to change into radium. Meyer and von Schweidler (Wien. Anzeiger, 1906, xii. Sitzung $\Lambda$ pril 26) have shown that such an extremely slight residual activity does exist, less than $1 / 10,000$ part of the initial excited activity, and the present experiment was tried to see if it is, or if it produces, radium, looked for as always by its characteristic emanation. One peculiarity this residual activity exhibits is that it decays rapidly, with a period of 11.7 days, which is difficult theoretically to account for. This experiment was carried out in a different laboratory to the others. 0.1 gram of actinium of activity about 300 times uranium was all that was available, and the first attempt to collect the residual activity from this met with an accident. A second was started on January 2nd, 1907. A loop of platinum wire mounted in glass tubes in a rubber cork, and held by the latter in a small brass cylinder containing the actinium in a platinum dish. The cylinder was connected to the positive pole and the wire to the negative pole of the 250 volt supply. The cylinder was closed airtight, and the experiment left for 160 days, when the wire was removed and examined. It was immediately inserted in a glass cylinder provided with two tubes prepared for it, the whole being closed air-tight.

After twenty-four hours the air was blown out and tested for the presence of the radium emanation in the electroscope, but no detectable amount was present. The wire was removed, and the residual activity in an electroscope produced a leak of $4 \cdot 6$. The wire was immediately returned to its glass case and left a week till June 9th, 1907, when it was again tested for the generation of radium emanation.

This time the wire was heated to dull redness in the dark without removal froin its glass case by means of a current. Again not the slightest appreciable leak of the electroscope occurred, although under the conditions of the test the presence of $2 \times 10^{-12}$ gram of radium could have been detected. The residual activity of the wire at this stage was $2 \cdot 9$, and eight days later 1.56 , which agrees roughly with Meyer and von Schweidler's statement. The wire has been again carefully closed up and will be tested at intervals for the generation of radium.

In connexion with this problem the results obtained by Boltwood ('Nature,' Nov. 15th, 1906, p. 54 ; Am. Journ. Sci. Dec. 1906, p. 537) and Rutherford ('Nature,'Jan. 17th, 1907 , p. 270; June 6th, 1907, p. 126) may be outlined. Boltwood separated the actinium present in a kilogram of carnotite as completely as possible and sealed it in a glass flask. In the course of 194 days the quantity of radium 
present had increased from $5 \cdot 7$ to $14 \cdot 2$ ( $\times 10^{-9}$ gram). Rutherford observed an increase in the amount of radium in an actinium solution corresponding to $3 \times 10^{-9}$ gram radium per year in 0.5 gram of actinium (activity 250 times uranium). He refers also to experiments witl the residual excited activity of actinium which was obtained from a very active actinium preparation, and dissolved off the platinum plate on which it was deposited by acid, and then tested for the growth of the radium emanation. He records a real growth of radium in the solution, which was much less than what is theoretically to be expected if actinium B changes directly into radium. In the later communication Rutherford finds thit the parent of radium can be separated from actinium and is a separate substance. He considers it not improbable that actinium may have no direct connexion with the parent of radium. If this is the case the residual activity of actinium should not generate radium.

\section{Experiment VI.}

If the early positive result was correct, the residues from the uranium should contain the radium-producing substance which has lately been found by Boltwood and Rutherford in preparations of actinium, and this substance should not be, at least wholly, removed by the old method of precipitation with barium sulphate. The residues wero therefore purified as carefully as possible from radium by this method. They contained between 100 and 200 grams of uranyl nitrate, anil were dissolved in about two litres of water containing excess of sulphuric acid. The liquid was precipitated in the cold four times, each with one gram and once with $2 \cdot 5$ grams of barium nitrate, the barium sulphate being removed after each addition. It was then evaporated to 300 e.c. and tested by the bubbling method, which showed that the quantity of radium present was still greater than $10^{-10}$ gram. The solution was again diluted and precipitated hot five times with one gram and once with two gratms of barium nitrate. It was finally sealed up in a glass flask provided with inlet and outlet tubes, sealed into the neck, on June 17th, 1907, and first tested on June 20th, 1907, in the manner described in the case of the samples of uraninite. No appreciable quantity of radium was present. The increase of the electroscope leak was only $0 \cdot 23$. A second test on July 3rd, 1907, in an electroscope giving $3 \cdot 1$ to the $\gamma$-ray test, gave a corrected equilibrium leak of $0 \cdot 33$, corresponding to the presence of only $4 \times 10^{-12}$ gram of radium.

It is hoped in the course of time that this experiment and 


\section{On the Relation between Uranium and Radium.}

others of similar character now in hand will throw further light upon the early positive result obtained by the bariumsulphate method of precipitation. But the following result may be cited. The two fifty gram samples of uranyl nitrate, one purified by barium sulphate and the other in the condition purchased, except that it is dissolved in water, which were used in the very first preliminary tests on April 1st and March 31st, 1904, respectively, have recently been tested, as before, by the bubbling method. They have been left in their original bottles, the tubes closed with rubber caps, and therefore no great precaution has been taken against the possibility of contamination with extraneous radium. They had not been opened for about three and a half years. Initially the leak from the purified sample corresponded to $\cdot 013$ of the present scale, and of the unpurified $0 \cdot 4$. Now the quantity of radium in the two bottles is indistinguishable by the rough bubbling test, and both give a leak by this test of from 1 to 2. In the old kilogram of uranyl nitrate a leak of about 15 was recorded after 550 days, corresponding to a leak of 2 for 50 grams in 1580 days. These two samples have now been sealed up in flasks and will be accurately tested for amount of radium as soon as they are ready. Even if they have been contaminated with radium, they will still furnish a valuable maximum limit of the rate of production of radium from commercial uranyl nitrate. The present rough results show that this is extremely slow, and that the radium present, with such small quantities of uranium, is even after four years not at all conspicuous. Nevertheless it agrees with the early result.

\section{Summary.}

(1) 250 grams of uranium (element) in the form of uranyl nitrate, purified by extraction with ether and containing initially $2 \cdot 3 \times 10^{-11} \mathrm{gram}$ of radium, has not increased in radium in 600 days by more than $10^{-11}$ gram.

(2) In another experiment with 481 grams of uranium containing initially only $1.3 \times 10^{-11}$ gram of radium the increase in amount of radium in 200 days is not greater than $5 \times 10^{-12} \mathrm{gram}$. Both this and the preceding experiment indicate that the production of radium is not greater than of the order of one ten-thousandth part of the amount of uranium disintegrating.

(3) In 278 grams of uranium purified by the same process there has been no appreciable grow th of actinium in the course of 300 days. 
(4) The residual activity of actinium is not due to radium and no appreciable growth of radium has yet occurred from it, although the activity has decayed to a small fraction of its initial value.

(5) An experiment to detect a possible gaseous intermediate product between uranium and radium has so far given a negative result.

(6) The residues from the purification of the uranium by ether have been very perfectly freed from radium by barium sulphate, and sealed up for future examination.

XXIII. Rays of Positive Electricity.

To the Editors of the Philosophical Magazine.

Gentlemen,-

Cavendish Laboratory, Oambridge. July 12 , 1907.

$\mathrm{T}$ his letter published in the June number of the Philo1 sophical Magazine, Professor W. Wien ascribes the constancy of the value of $e / m$ observed for Canalstrahlen in different gases to the presence of hydrogen as an impurity in the discharge-tube. The reasons which have led me to the conclusion that this explanation is not adequate are as follows. Although it is impossible to be sure that all the hydrogen has been eliminated from a discharge-tube, the amount present can be varied within very wide limits; and when this is done there is no corresponding variation in the brightness of the parts of the Canalstrahlen which are ascribed by Professor Wien to the presence of hydrogen. In all my experiments at low pressures, by far the greater part of the luminosity on the phosphorescent screen was produced by rays corresponding to values of $e / m$ equal to $10^{4}$ and $5 \times 10^{3}$, and this was equally the case whether the most elaborate precautions were taken to exclude hydrogen, or whether hydrogen was purposely introduced into the tube. I described in my paper an experiment where the tube was exhausted until the discharge would not pass; at this stage small quantities of one or other of the different gases, air, oxygen, carbonic oxide, helium, argon, neon, and hydrogen itself, were let into the tube through drying-tubes, so as to increase the pressure sufficiently to allow the discharge to pass, although it was still very small ; the appearance of the Canilstrahlen with equal currents through the tube was the same in all cases although the amount of hydrogen must bave varied enormously. In another experiment, made since the publication of my 